\title{
A Three Dimensional Grid Construction for the Simulation of Horizontal Wells in Geologically Complex Petroleum Reservoirs
}

\author{
AMADO, Luiz C.N., North Fluminense State University (UENF), Brazil, SPE member \\ HEINEMANN, Zoltán E., Leoben Mining University, Austria, SPE member
}

Paper presented at the 5th European Conference on the Mathematics of Oil Recovery, Leoben, Austria, 3-6 Sept. 1996

\begin{abstract}
Flexible gridding techniques have received great attention in the petroleum literature lately. Voronoi and other nonCartesian grids using control volume formulations have been investigated and have become common in reservoir engineering studies. Several grid geometries have been presented including orthogonal and non-orthogonal ones.
\end{abstract}

Nevertheless most of the different types of grids or combinations of them do not consider the well trajectory or anisotropies when modeling horizontal wells in geologically complex petroleum reservoirs. This paper describes a new method for modeling anisotropic reservoirs including the application to horizontal wells.

For a sub domain of the overall grid, a so-called "window", a three dimensional irregular mesh formed by an array of tetrahedrons is constructed around the horizontal well, using the k-orthogonal (or "general PEBI") approach. It takes not only the geometry of the well into consideration but also the anisotropic behavior of the permeability across the reservoir.

The control volume finite difference (CVFD) formulation is employed to discretize the fluid flow equations. The model is tested and validated by comparing the simulation results with analytical solutions, which are available for horizontal wells producing under different flow periods.

At the end of this paper some examples of general anisotropic reservoirs showing different orientations of the parameter permeability are presented in order to evaluate the applicability and usefulness of the method in modeling these occurrences.

\section{INTRODUCTION}

A method of constructing finite difference approximations for the quasi-linear Poisson's equation using non-uniform triangular mesh was first reported by Winslow [18]. This method was applied to solve magnetostatic problems and used the Median approach for the construction of the blocks. Amado and Pedrosa [1] introduced this type of gridding to reservoir simulation.

Many concepts have been developed recently concerning flexible gridding. The use of several grid systems independently or combination of some of them, as the locally refined systems, is receiving more and more attention. The locally refined systems use generally a Cartesian grid as an overall grid and another one as a sub domain in areas of particular interest, which is more complex and only defined locally.

Not only different types of grids but also different methods of discretization have been analyzed.

\section{CVFE Discretization Method}

The control volume finite element method (CVFE) is one of them. It allows us to integrate the fluid flow equations over irregular grid geometries, motivating the development of simulators which use or at least present non-Cartesian grids as an option for the user.

The control volume finite element method can be derived using the method of weighted residuals (MWR). The weighting function $w_{i}$ can be chosen in many ways. Each choice corresponds to a different criterion of MWR.

(i) If the weighting function is chosen to be the same as the trial functions, the Petrov-Galerkin finite element method is obtained.

(ii) If the weighting function is chosen to be one over the control volume and zero everywhere else, the CVFE is obtained.

Applying the CVFE for a typical porous media fluid flow 
equation, it is possible to get the expression below:

$$
\int_{c v} \frac{\partial}{\partial t}(\phi S) d V=\int_{c v} \nabla \cdot(\lambda k \nabla \Phi) d V
$$

which is the starting point for discretizing the conservation equation in the integral finite difference approach (IFD). In this work, the integral on the right side of equation (1) is calculated and evaluated as shown in the appendix at the end of this paper.

\section{Previous Work}

Heinemann and Brand [8] introduced the perpendicular bisection (PEBI) grid to simulate the flow of fluids in petroleum reservoirs, using the integral method of formulation for a block with any number of neighbors.

Forsyth [6] used the PEBI grid as an intermediate grid to improve the connection between coarse and fine Cartesian grids.

Palagi and Aziz [12] investigated the PEBI grid and the combination with other grids for field scale simulation and for the modeling of vertical and horizontal wells.

Pedrosa and Aziz [14] showed that the hybrid-Cartesian and hybrid-hexagonal grids are less sensitive to the grid orientation effects than the pure hexagonal or the 9-point scheme.

Patankar \& Baliga [11] made a lot of investigations using the control volume finite element method (CVFE). They employed interpolation functions as trial functions to solve mechanical problems in heat transfer and in fluid flow over a domain composed basically by PEBI or Median blocks.

In the following fig. 1 the 2D grid construction following the Median and the PEBI approach are shown:

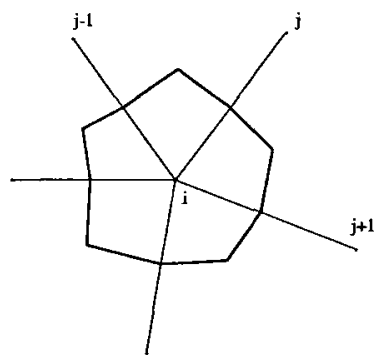

(a)

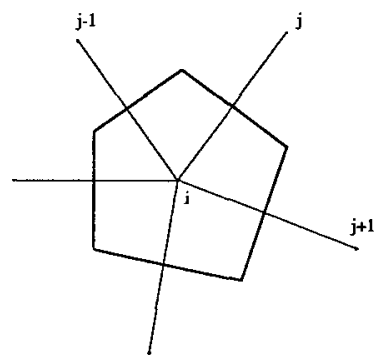

(b)
Fig. 1: (a) 2D Median approach; (b) 2D PEBI approach

Fung et al. [5] used also PEBI and Median grids with the control volume finite element method but applying the area coordinates for a linear triangular element as trial functions, as it is commonly used in finite elements formulations. They assumed an isotropic permeability field and positive transmissibility condition should be observed for some elements geometries and permeability combinations.
As pointed out by Heinemann et al. [7] the PEBI grid in anisotropic reservoirs cannot keep its shape and orthogonality because the unit vector, which is perpendicular to the block faces, is affected by the permeability.

If the permeability is treated as a tensor, the orientation between the gradient pressure vector (in the grid line direction) and the block faces is changed, loosing the orthogonality.

A full 3D k-orthogonal grid is constructed in such a way that this orthogonality is still kept. Heinemann et al. [7] introduced this concept and applied it to heterogeneous and anisotropic reservoirs with horizontal wells. Neither interpolation functions, which can be very complex in 3D domains, nor area coordinates with triangular elements are used.

Also, it seems to be reasonable to evaluate terms in the centroids of the blocks, especially when treating heterogeneities and anisotropies because different properties can be used in different parts of the same grid block.

Some authors [15],[16] have addressed the concept of treating permeability as a tensor and considered it to be one of the most appropriate ways to handle anisotropic cases. Depending on the type of the considered problem, this can lead to significant differences in the results.

In this work a full permeability tensor is incorporated into the transmissibility terms during the discretization process.

\section{THE K- ORTHOGONAL GRID}

In two dimensions, a network of triangles is formed and the block boundaries are constructed not by taking the perpendicular bisectors of each side of the triangles, but segments connecting the middle point of each side with a specified point within the triangle.

This point is determined in order to provide orthogonality when multiplied by the permeability tensor.

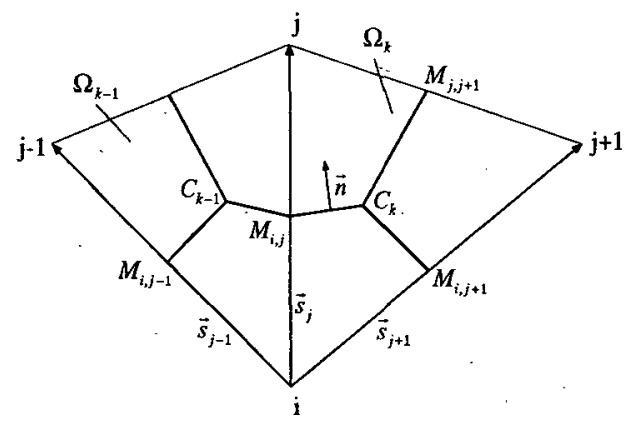

Fig. 2: $K$-orthogonal grid construction in $2 D$

In three dimensions, the network is an array of tetrahedrons. The k-orthogonal blocks are constructed in the same way: 


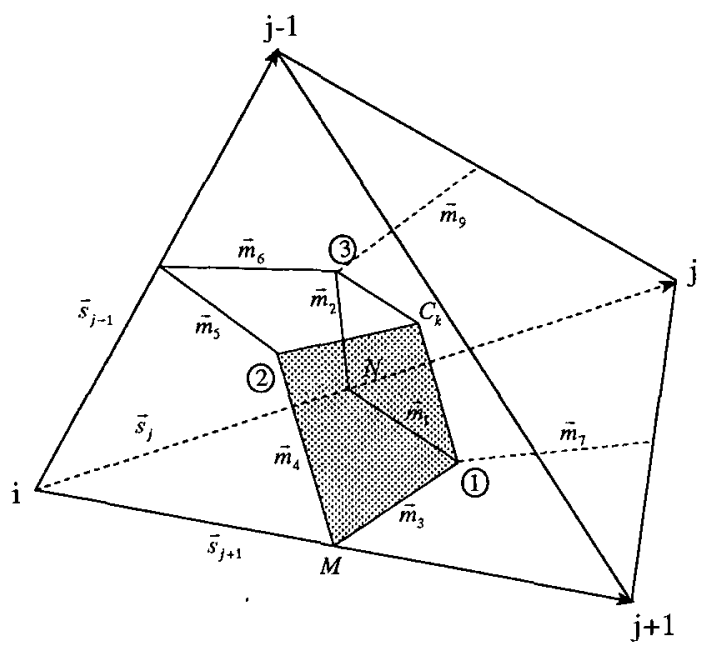

Fig. 3: K-orthogonal grid construction in 3D

The initial set of tetrahedrons is formed in such a way that all the 4 corner points are the closest ones in that space region and no other point falls in the region within the tetrahedron, defining a Delaunay Tesselation [17].

Once the grid points were located and the grid blocks formed, a set of material balance equations is written for each block, considering the flow through the blocks boundaries.

The discretization process is performed through the IFD (integral finite difference) method with some modifications for the transmissibility terms in order to include the full permeability tensor and the approximation for the scalar product, expressed by the unit normal vector (at the block boundaries) times the permeability tensor times the pressure gradient. This is performed at the centroid of the tetrahedrons and expanded as a function of the variables at the grids points.

The accumulation term is discretized as it is usually done in the integral method of discretization.

For sake of simplicity the mathematical model is presented in the appendix of this paper, where details are shown.

The discretization form of the fluid flow equations and the transmissibility coefficients are shown below:

$$
\begin{aligned}
& \sum_{j=1}^{N_{i}} T_{i j}\left(\frac{K_{r_{p}}}{\mu_{p} B_{p}}\right)_{i j}^{n+1}\left(\Theta_{p_{j}}-\Theta_{p_{i}}\right)^{n+1}= \\
& \frac{V_{b_{i}}}{\Delta_{t}}\left[\left(\frac{\phi S_{p}}{B_{p}}\right)_{i}^{n+1}-\left(\frac{\phi S_{p}}{B_{p}}\right)_{i}^{n}\right]+q_{p_{i}}^{n+1} \\
& T_{i j}^{(k)}=A_{i j}^{(k)} \frac{\left|k_{k} \vec{n}\right|}{\left|\vec{s}_{j}\right|}= \\
& =\frac{\left|\bar{k}_{k} \vec{n}\right| \cdot\left|\left(\vec{m}_{1}+\vec{m}_{2}\right) \times\left(\vec{c}_{k}-\frac{1}{2} \vec{s}_{j}\right)\right|}{2\left|\vec{s}_{j}\right|}
\end{aligned}
$$


finally an interpolation routine is applied.

The physical properties considered are permeability and porosity. The permeability is given as a tensor by the main permeability components, plus the unit vectors of the main permeability axes with respect to the $x, y, z$ axes of the basic block model. The porosity is also given for the basic blocks and interpolated for the window grid points.

Thickness and depth of the layers are not taken into consideration within the window because the windows blocks are irregular ones.

\section{WELL MODEL}

Well models for irregular blocks were developed by many authors for horizontal wells, using either Van Poolen's type or Peaceman's type.

The well model considered here is based on an analytical model in a similar way as described by Palagi and Aziz[13].

It is also possible to develop another model taking into consideration the wellbore effects and pressure drop in the wellbore. Then it is necessary to consider the wellbore as a grid block of the size of $r_{w}$.

\section{Horizontal Well}

The horizontal well analytical model was derived following the logic proposed by Palagi and Aziz. It takes into consideration the actual transmissibility coefficients and the anisotropic behavior of the permeability around the well.

If we consider a logarithmic pressure drop near the well, the pressure drop can be expressed by:

$$
p_{j}-p_{i}=\frac{Q B \mu}{\theta k_{i j} h_{i, i+1}} \ln \frac{d_{i j}}{r_{o}}
$$

and considering the flow configuration around the well:

$$
Q=\sum_{j=1}^{n} T_{i j}\left(p_{j}-p_{i}\right) \cdot \frac{1}{B \mu}
$$

Replacing the pressure drop in equation (5) by the expression given in equ.(4) yields:

$$
Q=\frac{Q}{\theta h_{i, i+1}} \cdot \sum_{j=1}^{n} \frac{T_{i j}}{k_{i j}} \ln \frac{d_{i j}}{r_{o}}
$$

and then the final expression for the apparent well block radius can be obtained as:

$$
\ln r_{o}=\left[\sum_{j=1}^{n} \frac{T_{i j}}{k_{i j}} \ln d_{i j}-\theta h_{i i+1}\right] \div \sum_{j=1}^{n} \frac{T_{i j}}{k_{i j}}
$$

The well index WI and the wellbore flowing pressure $p_{w f}$ are:

$$
\begin{aligned}
& W I=\left[\frac{\theta h_{i i+1} k_{a v}}{r_{o}}+s\right] \\
& q=-W I \frac{\lambda}{r_{w}}\left(p_{i}-p_{w f}^{*}\right) \\
& p_{w f}^{*}=p_{w f}+\rho_{a v} g\left(D_{i}-D_{r e f}\right)
\end{aligned}
$$

In the previous expressions, $k_{\mathrm{av}}$ is equal to $\sqrt{k_{\max } \times k_{\min }}$ while $k_{i j}$ are the permeabilities calculated along the grid lines.

$d_{i j}$ are the distances between the grid points $\mathrm{i}$ and its neighbor $\mathrm{j}$ while $h_{i i+1}$ represent the distances between the grid point $i$ and its neighbor $i+1$ in the wellbore.

$T_{i j}$ is the transmissibility to the neighboring block $\mathrm{j}$ and $r_{o}$ is the apparent well block radius.

As the well is situated inside the window area, the angle $\theta$ is taken equal $2 \pi$.

\section{Slanted Wells}

For inclined or slanted wells we use the same analytical model. The well trajectory is again represented by a set of points and the blocks are constructed in the same way around the well trajectory as described for the horizontal well.

\section{Wellbore Discretization}

The grid generation algorithm was built to place six points around the well in a certain distance, which can be specified by the user. These points are located in planes normal to the well trajectory.

With this method we can place points also in a circle at a distance $r_{w}$ from the wellbore. Then it is possible to construct the well blocks with the same size or very close to the size of the wellbore.

Many points can be placed at the well trajectory; usually they are placed regularly in equal distances.

\section{VALIDATION}

The results obtained with the 3D modeling grid and using the analytical model for the well are compared with those given by analytical solutions. A problem test, which is well known and is described by Babu and Odeh [2],[3] was chosen as reference run to validate the grid and the method proposed. The input data and a description of the problem are given in the appendix at the end of the paper. 
The three dimensional grid was set up as a sub domain in the middle of the reservoir with a horizontal well in the centre of the reservoir. An in-house simulator was used to perform the runs, with the 3D k-orthogonal grid implemented as a sub domain ("3D window").

The Cartesian grid was composed by $20 \times 10 \times 3$ blocks, the $3 \mathrm{D}$ window grid was composed by $6 \times 6 \times 12$ blocks.

The results of the simulation run were compared with those obtained with the analytical solution according to Babu and Odeh [2],[3] and Kuchuk et al. [10]. The figures 4 and 5 show the results for a whole range of time, from minutes to days, giving a broad overview of all the flow regimes occurring in that time.

For the early time period a logarithmic plot is used (fig.4); for the late time period a linear time scale is used (fig.5).

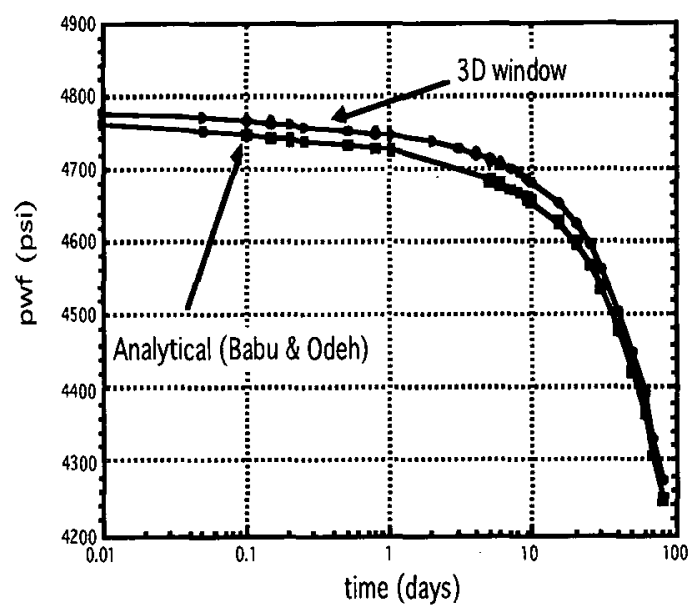

Fig. 4 : Validation - Early time

The agreement with the analytical solution shows that this grid is very suitable especially for the early time period (transient). Analyzing the results we can see that the agreement is very good for both flow regimes. Taking for instance the results for 0.01 days we get from the analytical solution $p_{w f}=4756.99 \mathrm{psi}$ and from the numerical simulation using the $3 \mathrm{D}$ window we get $\mathrm{p}_{\mathrm{wf}}=4759.75$ psi. After 0.1 days, the values are $4745.38 \mathrm{psi}$ and $4746.3 \mathrm{psi}$ respectively.

For the late time period the results are also correct as it can be seen in fig. 5 by the parallelism of the lines showing a difference to the analytical solution closely to 5 psi. E.g., taking the results after 20 days, we have $\mathrm{p}_{\mathrm{wf}}=4596 \mathrm{psi}$ (analytical) and $\mathrm{p}_{\mathrm{wf}}=4601 \mathrm{psi}$ (numerical). The differences between the average reservoir pressure $\mathrm{p}_{\mathrm{r}}$ and the bottom hole flowing pressure $\mathrm{p}_{\mathrm{wf}}$ is $82,38 \mathrm{psi}$. In the simulation run this difference was 4684.44 - 4601.52 $=82.92 \mathrm{psi}$.

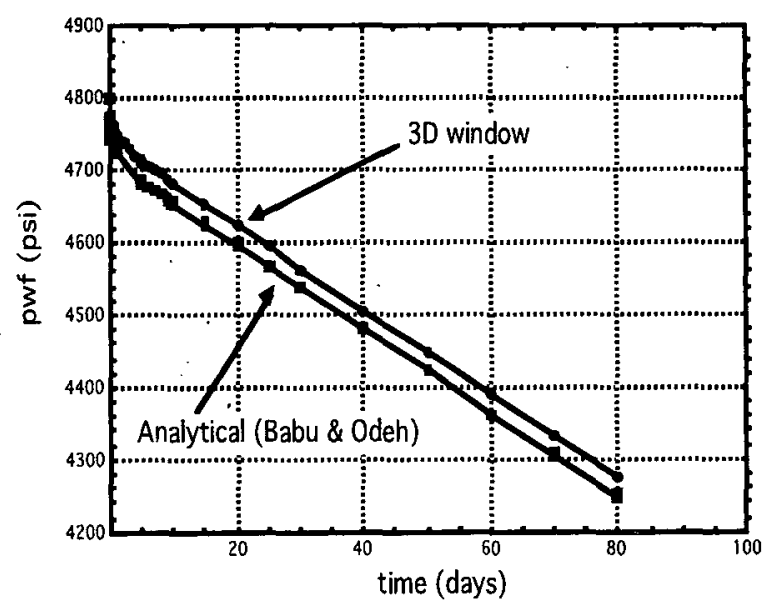

Fig. 5: Validation - Late time

As it could be observed, the 3D modeling seems to be very accurate for the simulation of the horizontal wells. It can be used for early and late time flow regimes, giving reasonable results for both.

\section{ANISOTROPY CONSIDERATIONS}

The anisotropy is investigated considering the permeability as a tensor evaluated at the centroids of the grid blocks. The input data are the permeabilities in the directions of the main permeabitilies axes and the correspondents unit vectors of those directions, given at the grid points of the underlying basic blocks.

Using these informations the permeabilities components $k_{i i}, k_{i j}$ are calculated by the following formulas and are inserted in the transmissibility coefficients.

$k_{x x}=k_{1} \cos ^{2} \theta_{r} \cos ^{2} \theta_{i}+k_{2} \sin ^{2} \theta_{r}+k_{3} \cos ^{2} \theta_{r} \sin ^{2} \theta_{i}$

$k_{z z}=k_{1} \sin ^{2} \theta_{i}+k_{3} \cos ^{2} \theta_{i}$

$k_{z z}=k_{1} \sin ^{2} \theta_{i}+k_{3} \cos ^{2} \theta_{i}$

$k_{x y}=k_{1} \sin \theta_{r} \cos \theta_{r} \cos ^{2} \theta_{i}$

$-k_{2} \sin \theta_{r} \cos \theta_{r}+k_{3} \sin \theta_{r} \cos \theta_{r} \sin \theta_{i}$

$k_{y z}=\left(k_{1}-k_{3}\right) \sin \theta_{r} \sin \theta_{i} \cos \theta_{i}$

$k_{x z}=\left(k_{1}-k_{3}\right) \cos \theta_{r} \sin \theta_{i} \cos \theta_{i}$

\section{APPLICATIONS}

In the following some applications concerning simulation of horizontal well using the 3D grid will be shown.

The 3D grid is constructed around the horizontal well as a window composed by a rectangular boundary as it is shown schematically by the shadowed region in fig. 6 , where different anisotropic regions with different orientations for the main permeabilities axes are also illustrated: 
2000.

1000.

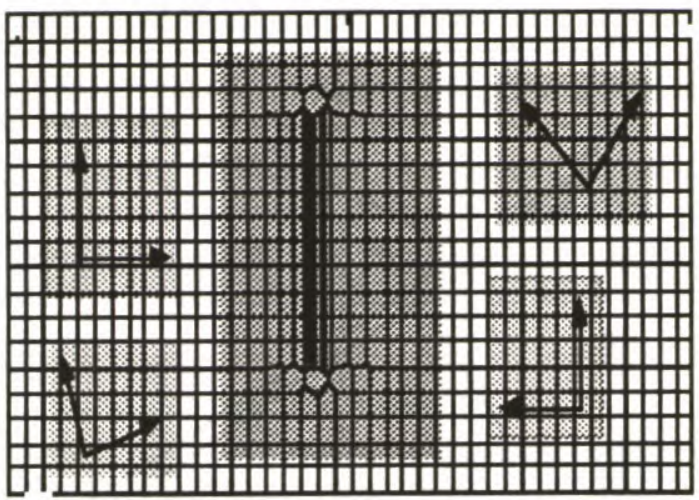

0.0

2000.

4000.

Fig. 6: Scheme of a $3 D$ window with anisotropic regions

Two different early flow periods which usually occur in horizontal wells are simulated: Early radial and early linear using examples from the literature [2],[3] for comparison of the results.

In order to investigate the behavior of anisotropic reservoirs two additional runs are also presented with different anisotropic ratios and orientations for the main permeabilities axes.

\section{Early radial and Early linear}

The early data time suggests that the flow can be early radial or early linear, depending on the geometry of the problem, reservoir properties, well length, and so on.

For the early radial period a drawdown test was run in a horizontal well. The well produced at a flow rate of 800 bbl / day and from a drainage volume $200 \mathrm{ft}$ thick, $4000 \mathrm{ft}$ long and $2000 \mathrm{ft}$ wide. The well length was $1000 \mathrm{ft}$. The window is constructed over an area corresponding to $6 \times 13 \times 3$ basic blocks. There are 16 well blocks regularly spaced along the well trajectory.

The pressure drop at the well $\mathrm{p}_{\mathrm{w}} \mathrm{f}(\mathrm{t})$ is given below, for the $3 \mathrm{D}$ window and for the respective analytical solution.

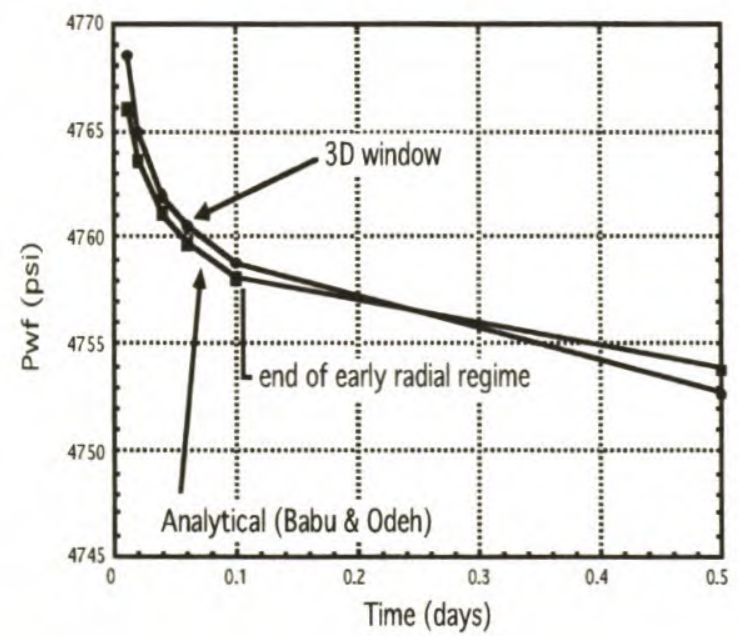

Fig. 7 : Early radial flow period
In the second example an early linear flow is expected to occur. A drawdown test is run on a horizontal well located in the center of a box-shaped drainage volume $100 \mathrm{ft}$ thick, $4000 \mathrm{ft}$ long and $4000 \mathrm{ft}$ wide. The well length was $2500 \mathrm{ft}$. The window is constructed this time over an area corresponding to $6 \times 16 \times 3$ basic blocks. There are 9 well blocks regularly spaced along the well trajectory.

The pressure drop at the well $p_{w f} v$ v. time is given below, for the 3D window and for the analytical solution.

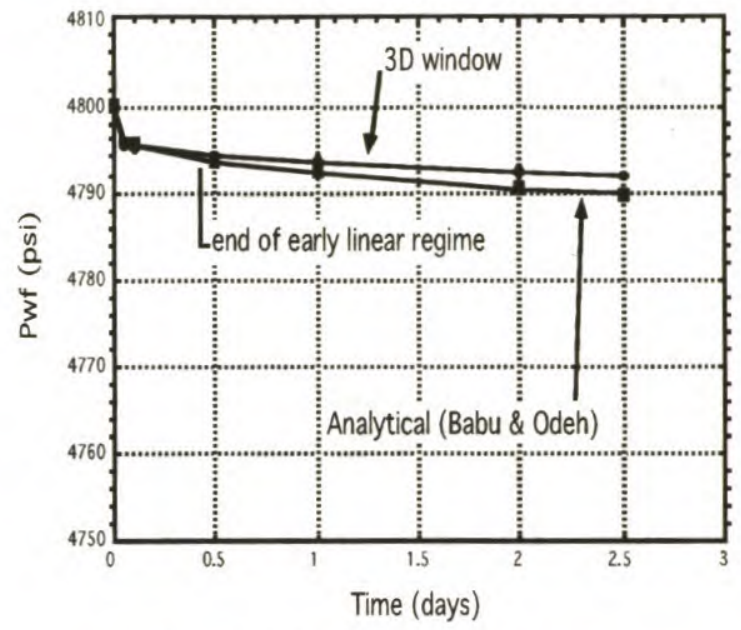

Fig. 8: Early Linear flow period

It is possible to observe that the agreement with the analytical solution is very good (the differences are less than $1 \mathrm{psi}$ ) during the whole early linear transient period.

After that the early linear regime is finished. Considering this the curves above show different slopes because this represents a transition from the transient flow to another one yet undefined at this time.

\section{Isotropic and Anisotropic cases}

The previous examples can be considered as isotropic ones because the permeability tensor was taken as a constant and diagonal matrix. Despite different values are used for the permeabilities in the $\mathrm{x}, \mathrm{y}$ and $\mathrm{z}$ directions, the tensor is always aligned with the main permeability axes. Except when $k_{i j}$ is different to zero, a pressure gradient along a coordinate direction will cause flow in an orthogonal direction.

In the following the example for the early radial period is simulated again but now using different angles for the main permeabilities axes. The results are always the same regardless the direction of the main permeabilities axes, as it would be expected for an isotropic reservoir: 


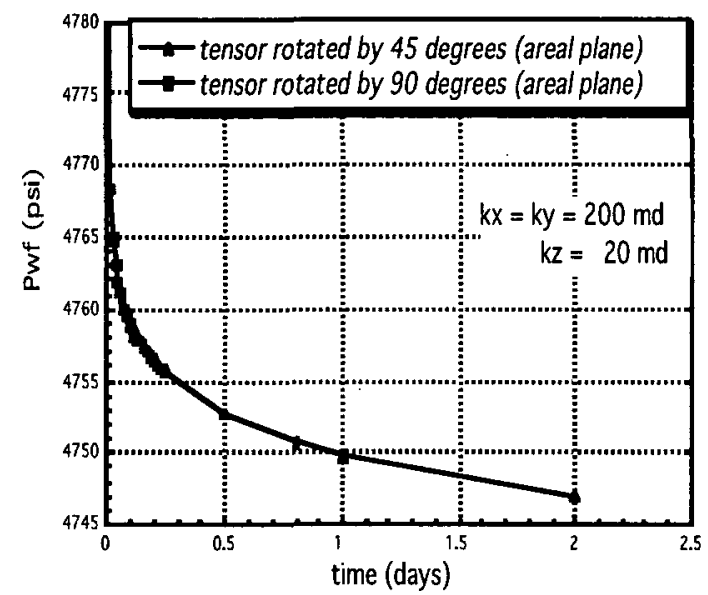

Fig. 9 : Isotropic reservoir in the areal plane

The second example presents different permeabilities in the areal plane and also in the vertical one. It is also possible to consider the effects of the reservoir boundaries because the simulation is run longer than before.

At first, the simulations are performed rotating the tensor only in the areal plane, by $45^{\circ}$ and $90^{\circ}$. Second, the areal orientation is kept constant and different inclinational angle are taken into consideration $\left(30^{\circ}\right.$ and $\left.45^{\circ}\right)$.

In the following the results for $p_{w f} v s$. time are shown:

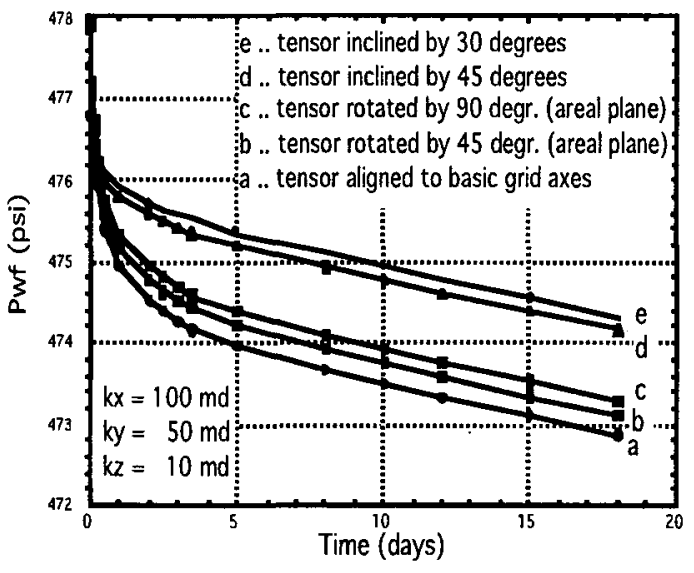

Fig. 10: Anisotropic reservoir

As it can be seen, the orientation of the permeability tensor is very important, especially when the anisotropy ratio is high. In this case small changes cause big differences. In this example for a factor of two between $\mathrm{k}_{\mathrm{x}}$ and $\mathrm{k}_{\mathrm{y}}$, no significant changes can be detected. For $\mathrm{a}$ factor of 10, however, (between $k_{x}$ and $k_{z}$ ) the differences are considerably greater.

\section{CONCLUSIONS}

This work describes a new gridding system to model geologically complex petroleum reservoirs considering horizontal wells in field scale simulation models. It honors not only the geometry of the well but also the anisotropic behavior of the permeabilities along the reservoir.

For a chosen part of the grid called "window", a locally full three dimensional irregular mesh formed by tetrahedrons is constructed. The grid blocks are obtained by the k-orthogonal method and linked to the conventional external grid.

The "window" is defmed as a sub-domain of the underlying basic Cartesian grid. It is limited by vertical planes and by the top and bottom surfaces. The vertical planes are defined by boundary points, specified previously, and the corresponding image points outside of the window which works as a linking between the two different grids.

Transient effects were intensively investigated under several circumstances and the results showed that the 3D modeling is capable of reproducing and matching this flow regime. This fact opens the possibility for important. future applications of this kind of grid, as for example its use in well testing analysis.

Anisotropies are addressed considering the parameter permeability as a full tensor. Examples showing different orientations for this parameter were presented in order to make comparisons between isotropic and anisotropic cases.

\section{ACKNOWLEDGEMEN'TS}

The authors would like to express their gratitude to the National Council for the Scientific and Technological Development of Brazil (CNPq) and to the "Österreichische Fonds zur Förderung der Wissenschaftlichen Forschung" for the support given to this research.

\section{NOMENCLATURE}

\section{Symbols:}

$A_{i j} \quad$ area of surface connecting blocks $i$ and $j\left[\mathrm{ft}^{2}\right]$

$B$ formation volume factor [bbl/STB]

$C_{k} \quad$ corner point inside the tetrahedron $\mathrm{C}_{\mathrm{k}}$

$\vec{c}_{k} \quad$ vector from gridpoint $i$ to corner point

$D$ top depth [ft]

$g$ gravity acceleration vector $\left[\mathrm{ft} / \mathrm{s}^{2}\right]$

$h$ thickness [ft]

$\bar{k}$ permeability tensor [Darcy]

$k_{i j} \quad$ components of permeability tensor [Darcy] 
$\vec{m}_{1} \quad$ vector connecting middlepoint $M_{i, j}$ with $C_{k}$

$M_{i j} \quad$ middle point between grid point $i$ and $j$

$\vec{n} \quad$ normal unit vector

$p \quad$ pressure [psi]

$p_{w f} \quad$ flowing well pressure [psi]

$\Delta \mathrm{p} \quad$ pressure difference [psi]

$Q \quad$ well flow rate $[\mathrm{bbl} / \mathrm{day}]$

$q_{p} \quad$ volumetric flow rate for phase $p[\mathrm{kmol} / \mathrm{s}]$

$r_{w} \quad$ wellbore radius [ $\mathrm{ft}$ ]

$r_{0}$ equivalent wellblock radius [ $\mathrm{ft}$ ]

$S_{p} \quad$ phase saturation [-]

$\vec{s}_{j} \quad$ vector connecting grid points $i$ and $j$

$T_{i j} \quad$ transmissibility

$\vec{u}_{p} \quad$ filtration velocity [ft/s]

$V_{i} \quad$ volume of gridblock $i\left[\mathrm{ft}^{3}\right]$

$x_{p c} \quad$ mole fraction of component $c$ in phase $p[-]$

$z$ depth [ft]

\section{Greek symbols:}

$\phi$ porosity [-]

$\Phi_{p} \quad$ phase potential [psi]

$\lambda_{p} \quad$ phase mobility [1/cp]

$\mu_{p} \quad$ phase viscosity [cp]

$\rho_{p} \quad$ phase density $\left[\mathrm{lb} / \mathrm{ft}^{3}\right]$

$\theta$ rotational angle

$\theta_{i} \quad$ inclinational angle

$\Omega_{k} \quad$ triangle joining gridpoints $i, j, j+I$

\section{Super/Subscripts:}

$P$

C

n

$n+1$

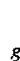

8

$\circ \quad$ oil

$p \quad$ phase

c component

i gridblock $I$

i adjacent gridblock $J$

$k$ grid element

w water

$x \quad \mathrm{x}$ direction

${ }_{y}^{x} \quad \mathrm{y}$ direction

\section{SI Metric Conversion Factors}

$\begin{array}{lll}b b l & \times 1.589873 & \mathrm{E}-01=m^{3} \\ c p & \times 1.0 & \mathrm{E}-03=P a s \\ f t & \times 3.048 & \mathrm{E}-01=m \\ f^{3} & \times 2.831685 & \mathrm{E}-02=m^{3} \\ l b / f t^{3} & \times 1.601846 & \mathrm{E}+01=k g / m^{3} \\ \text { Darcy } & \times 9.869233 & \mathrm{E}-12=m^{2} \\ p s i & \times 6.894757 & \mathrm{E}+03=P a\end{array}$

\section{REFERENCES}

[1] Amado, L.C.N. and Pedrosa, O.A.: "A Finite Volume Approach with Triangle Grids in Reservoir Simulation", paper SPE 23633, published in the SPE Advanced Technology Series, Vol.2, No.1, 179-185., March 1994

[2] Babu, D.K., Odeh, A.S.: "Productivity of a Horizontal Well", SPERE, Nov. 1989

[3] Babu, D.K., Odeh, A.S.,: "Transient Flow Behavior of Horizontal Wells: Pressure Drawdown and Buildup Analysis", SPEFE, March 1990

[4] Deimbacher, F.X. and Heinemann, Z.E.: "TimeDependent Incorporation of Locally Irregular Grids in Large Reservoir Simulation Models", paper SPE 25260 presented at the 1993 Symposium on Reservoir Simualtion New Orleans, Feb.28-Mar.3

[5] Fung, L.S.K., Hiebert, A.D. and Nghiem, L.: "Reservoir Simulation with a Control Volume Finite Element Method", paper SPE 21224 presented at 11th Reservoir Simulation Symposium, Anaheim, CA, Feb 17-20 1991

[6] Forsyth, P.A.Jr.: "A Control Volume Finite Element Method for Local Mesh Refinement," paper SPE 18415 presented at $10^{\text {th }}$ Reservoir Simulation Symposium, Houston, Feb. 6-8.1989

[7] Heinemann, Z.E., Amado; L.C.N., Ganzer, L.: "Finite Volume Discretization of the Fluid Flow Equations on General Perpendicular Bisection Grids", paper presented at the $5^{\text {th }}$ Intl. Forum on Reservoir Simulation, Muscat, Oman, Dec. 1994

[8] Heinemann, Z.E. and Brand, C.W.: "Gridding Techniques in Reservoir Simulation", Proc., First and Second Intl. Forums on Reservoir Simulation, Alpbach, Austria, $(1988,1989)$ 339-426.

[9] Heinemann, Z.E. and Deimbacher, F.X.: "Advances in Reservoir Simulation Gridding", paper presented at the $4^{\text {th }}$ Intl. Forum on Reservoir Simulation, Salzburg, Austria, Aug. 301992 
[10] Kuchuk, F.J. , Goode, P.A. , Brice, B.W., Sherrard, D.W. and Thambynayagam, R.K.M.: " Pressure Transient Analysis and Inflow Performance for Horizontal Wells", paper SPE 18300, 1990

[11] Patankar and Baliga, B.R.: "Some Recent Advances in Computational Heat Transfer and Fluid Flow", ENCIT 90, Itapema-SC (Brazil), Dec. 10-12 1990

[12] Palagi, C.L. and Aziz, K.: "Use of Voronoi Grid in Reservoir Simulation", paper SPE 22889 presented at the 1991 SPE Annual Technical Conference and Exhibition, Dallas, Oct. 6-9

[13] Palagi, C.L. and Aziz, K.: "The Modeling of Vertical and Horizontal Wells with Voronoi Grid", paper SPE 24072 presented at the 1992 SPE Western Regional Meeting, Bakersfield, CA, Mar.30-Apr.1

[14] Pedrosa, O.A. and Aziz, K.: "Use of a Hybrid Grid in Reservoir Simulation", SPERE , Nov. 1986, 61121; Trans., AIME, 282.

[15] Shiralkar, S. G.: " Reservoir Simulation of Generally Anisotropic Systems", paper published in the SPERE, Aug. 1990

[16] Wang, J.T.:" The Use of Permeability Tensors in Modelling Heterogeneous and Fractured Flow Media", paper presented at the EUROPEC-Student Paper Contest, Nov. 1992

[17] Watson, D.F.: "Computing the N-dimensional Delaunay Tesselation with Application to Voronoi Polytopes", Computer Journal, 24, 167-172., 1981

[18] Winslow, A.M.: "Numerical Solution of the Quasilinear Poisson Equation in a Nonuniform Mesh", J.Comp.Phys., 1967

\section{APPENDIX}

\section{Validation - Description of the example}

To show the efficiency of the constructed 3D window one example described by Babu and Odeh was chosen.

It consists of an horizontal well $1000 \mathrm{ft}$ long drilled in a box-shaped drainage volume $4000 \mathrm{ft}$ long, $2000 \mathrm{ft}$ wide and $100 \mathrm{ft}$ thick. The well lies between $\mathrm{y}_{1}=750 \mathrm{ft}$ and $\mathrm{y}_{2}$ $=1750 \mathrm{ft}$. The location in the $\mathrm{x}$ and $\mathrm{z}$ direction is defined by $x_{0}=3000 \mathrm{ft}$ and $z_{\mathrm{p}}=50 \mathrm{ft}$. The well is centrally located with respect to the $\mathrm{z}$ direction. The well radius is $0.25 \mathrm{ft}$ and the well produces at a flow rate of 2000 bbl/day with an initial pressure of $4800 \mathrm{psi}$ (reservoir initial pressure).
The reservoir is an anisotropic one with the permeabilities in the $\mathrm{x}, \mathrm{y}$ and $\mathrm{z}$ directions equal to $200 \mathrm{md}, 200 \mathrm{md}$ and $50 \mathrm{md}$, respectively.

The fluid properties are the following: viscosity $\mu=1 \mathrm{cp}$, total compressibility $c_{T}=1.5 \mathrm{E}-05$ and volume formation factor $\mathrm{B}_{\mathrm{o}}=1.25$.

\section{Discretization on General 3D Grid}

We need an approximation for the flow rates across the interfaces of the polygonal blocks constructed by the $\mathrm{k}$ -orthogonal method and considering the permeabilty as a full tensor.

Considering one phase flow of an incompressible fluid, the flow rate across any specified block interface can be expressed as :

$$
\begin{gathered}
Q_{k}=-\int_{A_{1 k}} \lambda_{p} \bar{k} \nabla \Phi \cdot \vec{n} d A-\int_{A_{2 k}} \lambda_{p} \bar{k} \nabla \Phi \cdot \vec{n} d A \\
-\int_{A_{3 k}} \lambda_{p} \bar{k} \nabla \Phi \cdot \vec{n} d A
\end{gathered}
$$

with: $\quad Q_{p_{i, j, k+1 / 2}}$ flow rate through the surface A

$$
\begin{array}{ll}
\lambda_{p} & \text { phase mobility } \\
\bar{k}^{2} & \text { permeability tensor } \\
\vec{n} & \text { normal vector to the surface A } \\
\nabla \Phi_{p} & \text { potential gradient }
\end{array}
$$

The integral over the area is approximated numerically taking $\nabla \Phi_{p}$ constant evaluated at the centroid of the tetrahedron and performing the integration over the areas $A_{1}, A_{2}$ and $A_{3}$. Explaining for a generic area $A$, we get:

$$
Q_{p_{i, j, k+1 / 2}}=-\lambda_{p_{i, j, k+1 / 2}}\left(\bar{k} \cdot \nabla \Phi_{p_{i, j, k+1 / 2}}\right) \vec{n} A
$$

The term $\vec{n} A$ can be substituted by the vectorial product of the vector $h$ times the vector $m$, as it was defined in the figure 3 in the beginning of the paper.

$$
\begin{array}{r}
\vec{n} A=\frac{1}{2}\left(\vec{h}_{1} \times \vec{m}_{1}+\vec{h}_{2} \times \vec{m}_{2}+\vec{h}_{1} \times \vec{m}_{3}+\right. \\
\left.\vec{h}_{3} \times \vec{m}_{4}+\vec{h}_{2} \times \vec{m}_{6}+\vec{h}_{3} \times \vec{m}_{5}\right)
\end{array}
$$

h...... is the segment which connects the centroid of the tetraedron and the centroid of the faces.

$\mathrm{m}$.... is the segment which connects the centroid of the faces and the middle point of the side

$\times \ldots$. is the vectorial product of the two vectors. 
As displayed in fig. 3 the following relationships are valid:

$$
\begin{aligned}
& \vec{m}_{1}+\vec{m}_{3}=\frac{1}{2}\left(\vec{s}_{j+1}-\vec{s}_{j}\right) \\
& \vec{m}_{6}+\vec{m}_{2}=\frac{1}{2}\left(\vec{s}_{j}-\vec{s}_{j-1}\right) \\
& \vec{m}_{5}+\vec{m}_{4}=\frac{1}{2}\left(\vec{s}_{j+1}-\vec{s}_{j-1}\right)
\end{aligned}
$$

Inserting equ.(A-4) and (A-3) into equation (A-2) yields:

$$
\begin{array}{r}
Q_{k}=\bar{k}_{k} \nabla \Phi_{k} \cdot \frac{1}{4} \cdot\left(\vec{h}_{1} \times\left(\vec{s}_{j+1}-\vec{s}_{j}\right)\right)+ \\
\bar{k}_{k} \nabla \Phi_{k} \cdot \frac{1}{4} \cdot\left(\vec{h}_{2} \times\left(\vec{s}_{j}-\vec{s}_{j-1}\right)\right)+ \\
\bar{k}_{k} \nabla \Phi_{k} \cdot \frac{1}{4} \cdot\left(\vec{h}_{3} \times\left(\vec{s}_{j+1}-\vec{s}_{j-1}\right)\right)
\end{array}
$$

The derivatives of the potential in the directions $\vec{s}$ will be calculated as :

$$
\begin{gathered}
\Phi_{j}-\Phi_{i}=\nabla \Phi_{k} \cdot \vec{s}_{j} \\
\Phi_{j+1}-\Phi_{i}=\nabla \Phi_{k} \cdot \vec{s}_{j+1} \\
\Phi_{j-1}-\Phi_{i}=\nabla \Phi_{k} \cdot \vec{s}_{j-1}
\end{gathered}
$$

The approximations used in the three equations (A-6), (A-7) and (A-8) are of the order of magnitude of $\Delta s_{j}, \Delta s_{j+1}$ and $\Delta s_{j+1}$, respectively.

Adding equ.(A-6) multiplied with $\vec{s}_{j+1}$, equ.(A-7) multiplied with $\vec{s}_{j-1}$ and equ.(A-8) multiplied with $\vec{s}_{j}$ and then solving for $\nabla \Phi_{k}$ yields:

$$
\begin{aligned}
\nabla \Phi_{k}= & \frac{\left(\Phi_{j}-\Phi_{i}\right) \vec{s}_{j+1}}{\vec{s}_{j} \cdot \vec{s}_{j+1}+\vec{s}_{j+1} \cdot \vec{s}_{j-1}+\vec{s}_{j-1} \cdot \vec{s}_{j}} \\
& \frac{\left(\Phi_{j+1}-\Phi_{i}\right) \vec{s}_{j-1}}{\vec{s}_{j} \cdot \vec{s}_{j+1}+\vec{s}_{j+1} \cdot \vec{s}_{j-1}+\vec{s}_{j-1} \cdot \vec{s}_{j}} \\
& \frac{\left(\Phi_{j-1}-\Phi_{i}\right) \vec{s}_{j}}{\vec{s}_{j} \cdot \vec{s}_{j+1}+\vec{s}_{j+1} \cdot \vec{s}_{j-1}+\vec{s}_{j-1} \cdot \vec{s}_{j}}
\end{aligned}
$$

Substituting equ. (A-9) in equ.(A-5) and rearranging:

$$
\begin{aligned}
Q_{k}= & \frac{1}{4 w} \cdot\left[\left(\vec{k} \vec{s}_{j+1}\right) \vec{u}\left(\Phi_{j}-\Phi_{i}\right)\right]+ \\
& \frac{1}{4 w} \cdot\left[\left(\bar{k} \vec{s}_{j-1}\right) \vec{u}\left(\Phi_{j+1}-\Phi_{i}\right)\right]+ \\
& \frac{1}{4 w} \cdot\left[\left(\vec{k}_{j}\right) \vec{u}\left(\Phi_{j-1}-\Phi_{i}\right)\right]
\end{aligned}
$$

The equation (A-10) can be written in the following shorter form:

$$
\begin{array}{r}
Q_{k}=a_{k} \cdot\left(\Phi_{j}-\Phi_{i}\right)+b_{k}\left(\Phi_{j+1}-\Phi_{i}\right) \\
+c_{k}\left(\Phi_{j-1}-\Phi_{i}\right)
\end{array}
$$

where:

$$
\begin{aligned}
a_{k}= & \frac{1}{4 w}\left(\vec{k}_{j+1}\right) \vec{u} \\
b_{k}= & \frac{1}{4 w}\left(\vec{k}_{j-1}\right) \vec{u} \\
c_{k}= & \frac{1}{4 w}\left(\vec{k} \vec{s}_{j}\right) \vec{u} \\
w= & \vec{s}_{j} \cdot \vec{s}_{j+1}+\vec{s}_{j+1} \cdot \vec{s}_{j-1}+\vec{s}_{j-1} \cdot \vec{s}_{j} \\
\vec{v}= & \vec{h}_{1} \times\left(\vec{s}_{j+1}-\vec{s}_{j}\right)+\vec{h}_{2} \times\left(\vec{s}_{j}-\vec{s}_{j-1}\right)+ \\
& \vec{h}_{3} \times\left(\vec{s}_{j+1}-\vec{s}_{j-1}\right)
\end{aligned}
$$

For an isotropic case the equations (A-12) can be written in a simpler form because two terms of the vector $\vec{v}$ are orthogonal to the corresponding $\vec{s}$, therefore:

$$
\begin{aligned}
& a_{k}=\frac{k}{4 w} \vec{s}_{j+1} \vec{h}_{2} \times\left(\vec{s}_{j}-\vec{s}_{j-1}\right) \\
& b_{k}=\frac{k}{4 w} \vec{s}_{j-1} \vec{h}_{1} \times\left(\vec{s}_{j+1}-\vec{s}_{j}\right) \\
& c_{k}=\frac{k}{4 w} \vec{s}_{j} \vec{h}_{3} \times\left(\vec{s}_{j+1}-\vec{s}_{j-1}\right)
\end{aligned}
$$

The flow rate across the boundaries for the grid block is obtained by summing up equ. (A-11) for each tetrahedron. Since the sum is cyclic, the discretized form of the flow terms becomes

$$
\sum_{j=1}^{n_{i}} Q_{i, j}=\sum_{j=1}^{n_{i}}\left(\sum_{k=1}^{m_{j}} a_{k}\right)\left(\Phi_{j}-\Phi_{i}\right)=0
$$

$Q_{i, j}$ can be interpreted as the flow rate between the blocks $i$ and $j$ (along grid line $i j$ ) and may be written as

$$
Q_{i, j}=T_{i, j}\left(\Phi_{j}-\Phi_{i}\right)
$$

$T_{i, j}$ is called block pair transmissibility for the block pair $i, j$. It is the same if calculated from both directions:

$$
T_{i, j}=T_{j, i}
$$

